\title{
Neonatal Encephalopathy: Association of Cytokines with MR Spectroscopy and Outcome
}

\author{
AGNES I. BARTHA, AUDREY FOSTER-BARBER, STEVEN P. MILLER, DANIEL B. VIGNERON, \\ DAVID V. GLIDDEN, A. JAMES BARKOVICH, DONNA M. FERRIERO
}

\author{
Department of Neurology [A.I.B., A.F.B., S.P.M., A.J.B., D.M.F.], Department of Pediatrics [S.P.M., \\ A.J.B., D.M.F.], Department of Radiology [D.B.V., A.J.B.], Department of Epidemiology and Biostatistics \\ [D.V.G.], University of California, San Francisco, San Francisco, California 94143
}

\begin{abstract}
In term neonatal encephalopathy, little is known about the relationship between early inflammatory markers, neonatal brain injury, and long-term neurodevelopmental outcome. Our goal was to determine whether neonatal serum cytokine levels are associated with cerebral metabolism assessed by proton magnetic resonance spectroscopy (MRS), with magnetic resonance imaging (MRI) abnormalities, and with neurodevelopmental outcome at 30 mo of age. Levels of seven cytokines [IL-1 $\beta$, IL-6, IL-8, IL-9, IL-12, IL-13, and tumor necrosis factor (TNF)- $\alpha$ ] were measured in dried neonatal blood by immunoaffinity chromatography in a prospective cohort of 62 term newborns at risk of neonatal encephalopathy. MR images $(n=61)$ were scored and lactate/choline and $\mathrm{N}$-acetyl-aspartate (NAA)/choline were measured by MRS $(n=42)$ on median day of life 6 in the deep gray nuclei (DGN) and in the watershed/cortical zone (WS). Neurodevelopmental outcome ( $n=54$ ) was considered abnormal if the infant died or if cognitive delay and/or functional motor deficit were detected at 30 mo. IL-1 $\beta$, IL-6, IL- 8 and TNF- $\alpha$ were significantly associated with lactate/choline in the DGN ( $p=$ $0.03,0.02,0.03$, and 0.01 respectively), but not in the WS (all $p$ $>0.1$ ). Cytokines were not associated with NAA/choline in any region or with MRI scores. Children with abnormal neurodevel-
\end{abstract}

\section{ABSTRACT}

opmental outcome had higher neonatal levels of IL-1 $\beta$, IL-6, IL-8, and lower levels of IL-12 $(p=0.04,0.03,0.01,0.03$ respectively). Elevated inflammatory cytokines were associated with impaired cerebral oxidative metabolism, but not with detectable MRI changes in the neonatal period. Understanding the link between elevated cytokines and outcome would inform novel strategies of cerebral protection. (Pediatr Res 56: 960966, 2004)

DGN, deep gray nuclei

ECMO, extracorporeal membranous oxygenation

${ }^{1} \mathbf{H}-\mathbf{M R S}$, proton magnetic resonance spectroscopy

MDI, Mental Development Index

MRI, magnetic resonance imaging

NAA, $N$-acetylaspartate

NE, neonatal encephalopathy

NMS, neuromotor score

RANTES, "regulated on activation, normal t-cell expressed and secreted" chemokine

TNF- $\alpha$, tumor necrosis factor- $\alpha$

WS, watershed/cortex
Recent evidence suggests that inflammatory cytokines play a role in the pathogenesis of NE. Elevated levels of IL-6 and IL-8 in the cerebrospinal fluid of term newborns have been correlated with the more severe encephalopathy (1). In term neonates born to mothers with clinical chorioamnionitis, the highest levels of IL-6, IL-8, and RANTES were measured in neonates who had NE and/or seizures (2). Furthermore, the neonatal inflammatory response may also influence neurodevelopmental outcome, as elevated cytokines in term newborns with NE correlate with early outcome (1). Neonatal serum

Received March 30, 2004; accepted July 7, 2004.

Correspondence: Donna M. Ferriero, M.D., Professor of Neurology and Pediatrics, Department of Neurology, University of California, San Francisco, 521 Parnassus Ave., C-215, San Francisco, CA 94143-0663; e-mail: dmf@itsa.ucsf.edu

Supported by National Institutes of Health Grants RR 01271, NS 35902, and NS 40117.

DOI: 10.1203/01.PDR.0000144819.45689.BB cytokines (IL-1, IL-8, IL-9, TNF- $\alpha$, and RANTES) were also significantly higher in term infants who later developed cerebral palsy $(\mathrm{CP})(3,4)$.

However, the association of cytokines with neonatal brain metabolism, brain injury, and longer-term cognitive and motor outcome in term NE has not yet been determined. The objective of this cohort study was to determine whether neonatal serum cytokine levels are associated with 1) measures of cerebral metabolism assessed by ${ }^{1} \mathrm{H}-\mathrm{MRS}$, 2) abnormalities consistent with brain injury on MRI, and 3) neurodevelopmental outcome assessed by developmental and neuromotor evaluations at 30 mo of age.

\section{METHODS}

Subjects. This cohort was derived from a prospective study designed to ascertain the MRI predictors of neurodevelopmen- 
tal outcome in term and near-term newborns at risk of NE. Consecutive newborns admitted to the Intensive Care Nursery at UCSF and an affiliated county hospital were screened between October 1993 and January 2000 to enroll 125 newborns with at least one of the following: 1) umbilical artery $\mathrm{pH}<7.1$; 2) umbilical artery base deficit $>10$; 3 ) Apgar score $\leq 5$ at 5 min of life; or 4) overt NE as assessed by a neonatologist (Fig. 1). Newborns were excluded if their gestational age was $<36$ wk, or if they had major congenital malformations, metabolic diseases, or infections (toxoplasmosis, other agents, rubella, cytomegalovirus, herpes simplex (TORCH). The protocol was approved by the Committee for Human Research at the University of California San Francisco and voluntary informed consent was obtained from parents or legal guardians. Following enrollment, all families were approached for additional consent to participate in the cytokine study. For the 72 newborns enrolled in the cytokine study, 62 stored neonatal blood samples were available for analysis of cytokine levels, making up the cohort presented here (Fig. 1).

Cytokines. Neonatal blood levels of inflammatory cytokines were measured using previously established methodology $(3,5)$. Heel-stick blood was drawn in all newborns on the first days of life as part of California's mandatory newborn screen for genetic diseases. All newborns requiring ECMO had their blood samples obtained before initiation of this therapy. Spots of whole blood were dried on Guthrie card filter papers, then preserved at $-26^{\circ} \mathrm{C}$ at the Genetic Disease Branch of the California Department of Health Services. After obtaining approval from the California Committee for the Protection of Human Subjects, we retrieved blood spots from 62 subjects. Those processing the blood spots were blinded to the subjects' clinical history. After processing, the protein content of each sample (equivalent to $15 \mu \mathrm{L}$ of whole blood) was measured spectrophotometrically at $260 / 280 \mathrm{~nm}$. The eluates were derivatized with the fluorochrome Cy5 (Research Organics, Cleveland, $\mathrm{OH}$ ) and the volume was adjusted to normalize the protein content to $1 \mu \mathrm{g} / \mathrm{mL}$ before injection into the analytical system. The samples were analyzed by recycling immunoaffinity chromatography as described (5). Twenty-five-microliter samples were injected into serially connected microcolumns, each column containing a different immobilized capture antibody. As the capture antibodies retained their specific analyte, the remainder of the sample passed on to the subsequent columns. The analytes were released by treatment with acidic buffer, then measured by laser-induced fluorescence detection. The analysis included IL-1 $\beta$, IL-6, IL-8, IL-9, IL-12, IL-13, and TNF- $\alpha$.

Neonatal condition. Clinical chart review included information relevant to the analysis of cytokine levels: placental or neonatal infection, neonatal blood transfusions, ECMO treatment, or maternal steroids, and score for neonatal acute physiology-perinatal extension (SNAP-PE) as an overall measure of systemic illness severity in the first $24 \mathrm{~h}$ of admission.

Magnetic resonance data. Of the 62 newborns in the cytokine study, 61 were studied with brain MRI; 1 newborn was clinically unstable for imaging. Of these 61 newborns, 42 had ${ }^{1} \mathrm{H}$-MRS performed as well $\left({ }^{1} \mathrm{H}-\mathrm{MRS}\right.$ was introduced to the study protocol in 1995) (Fig. 1). The same MR scanner and techniques were used for the entire cohort. The newborns were imaged at a median of $6 \mathrm{~d}$ of life (range, 2-16 d), as soon as they were clinically stable to be transported safely to the MR scanner and imaging time was available.

MRI of the brain in all newborns included $4 \mathrm{~mm}$ (1 mm gap) sagittal and axial spin echo images [500/11/2 repetition time (TR)/echo time (TE)/excitations], and $4 \mathrm{~mm}$ ( $2 \mathrm{~mm}$ gap) axial spin echo images $(3,000 / 60,120 / 1)$ through the entire brain. MRI abnormalities on the T1- and T2-weighted sequences were scored using a system previously described for the determination of neuromotor outcome (6) in the DGN as follows: 0 $=$ normal DGN or isolated focal cortical infarct; $1=$ abnormal signal in thalamus; $2=$ abnormal signal in thalamus and lentiform nucleus; 3 = abnormal signal in thalamus, lentiform nucleus, and perirolandic cortex; and $4=$ more extensive involvement. Findings in the WS were scored as follows: $0=$ normal WS; 1 = single focal infarction; 2 = abnormal signal in anterior or posterior watershed white matter; $3=$ abnormal signal in anterior or posterior watershed cortex and white matter; $4=$ abnormal signal in both anterior and posterior watershed zones; and $5=$ more extensive cortical involve-

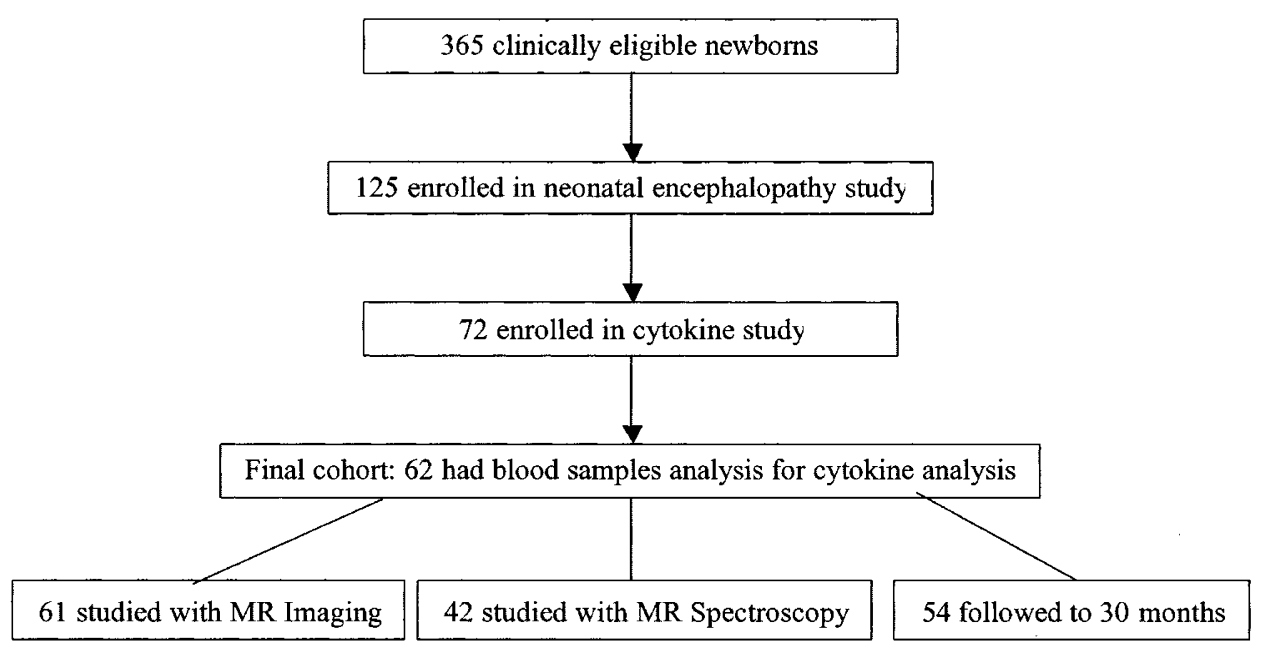

Figure 1. Flowchart illustrating cohort formation. 
ment. The maximum score on any sequence for both regions was used for the analysis.

${ }^{1} \mathrm{H}$-MRS may be used to detect elevation in lactate levels (lactate/choline), indicating disturbance in cerebral oxidative metabolism, and diminution in NAA levels (NAA/choline), indicating delayed cellular maturation, cellular damage, dysfunction, or death (7). Proton spectra were acquired from the DGN and frontal watershed zone (5 $\mathrm{cm}^{3}$ voxels) (Fig. 2) as these regions are particularly vulnerable to injury in the term newborn (8). ${ }^{1} \mathrm{H}$-MRS was performed using the point-resolved spectroscopy (PRESS) technique to acquire long echo time spectra with the PROBE (PROton Brain Exam; General Electric Medical Systems, Milwaukee, WI) sequence in under 5 min with $\mathrm{TR}=2 \mathrm{~s}$, TE $=288 \mathrm{~ms}$, and a total of 128 acquisitions, as previously described (9). After acquisition, the ${ }^{1} \mathrm{H}-\mathrm{MRS}$ data were transferred off-line and analyzed on a SPARC workstation (Sun Microsystems, Mountain View, CA). The ${ }^{1} \mathrm{H}-\mathrm{MRS}$ data were Fourier transformed and baseline fitted. The peak areas were integrated for the choline, creatine, NAA, and lactate resonances.

Developmental examinations. Pediatric neurologists blinded to the neonatal information assigned a NMS at standardized exams: $0=$ normal; $1=$ abnormal tone or reflexes or primitive reflexes; $2=$ abnormal tone and reflexes; $3=$ decreased power and tone or reflex abnormality; $4=$ cranial nerve involvement and any motor abnormality; $5=$ cranial nerve involvement and spastic quadriparesis; and $6=$ death (10). Cognitive development was satisfactorily scored for each child using the MDI of the Bayley Scales of Infant Development II test (11). Twelve-month $(n=60)$ and 30-mo $(n=54)$
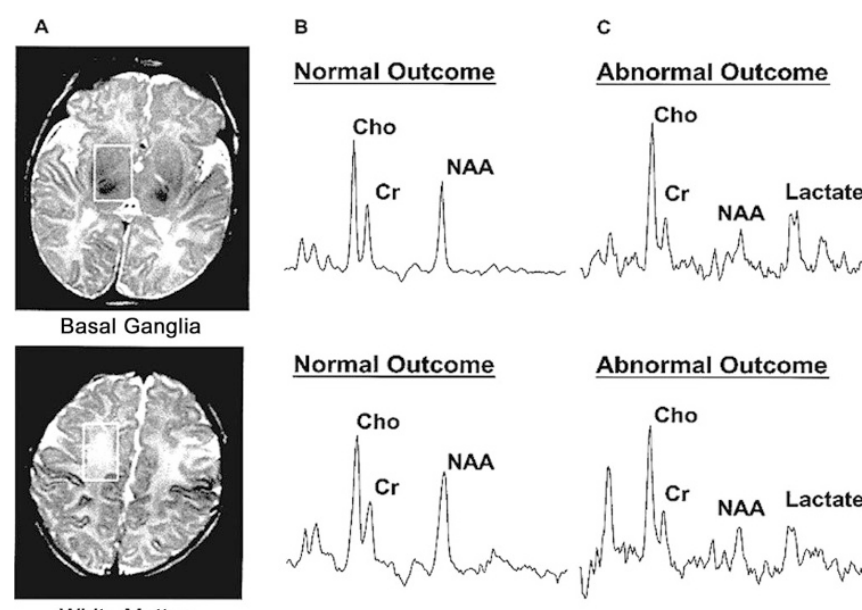

Normal Outcome

Abnormal Outcome

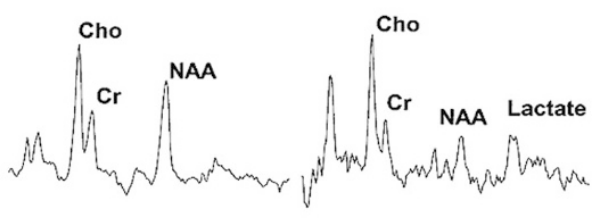

Figure 2. (A) Location of 1H-MRS voxels superimposed on axial T2 brain MR images of a term neonate (boxes). The DGN voxel includes lentiform nuclei, ventrolateral thalamus, and posterior limb of the internal capsule. The WS voxel includes primarily subcortical white matter in the region between the anterior cerebral artery and middle cerebral artery distributions. (B) Representative proton spectra of a newborn with normal neurodevelopmental outcome (voxels as shown, PRESS techniques TR $2 \mathrm{~s}$, TE $288 \mathrm{~ms}$, and 128 acquisitions). Note the absence of a lactate peak, and also that the amplitude of the NAA peak is intermediate between that of the choline ( $\mathrm{Cho}$ ) and creatine $(\mathrm{Cre})$ peaks. $(C)$ Representative proton spectra of a newborn with abnormal neurodevelopmental outcome (voxels as shown, PRESS techniques TR 2 s, TE 288 $\mathrm{ms}$, and 128 acquisitions). Note the presence of a lactate doublet, and the relative diminution of the NAA peak compared with the spectrum in $B$. neurodevelopmental outcome was classified a priori as functionally normal if the MDI $\geq 70$ and the neuromotor score $<3$ (no functional abnormality of motor power). Outcome was abnormal if the infant died, if the NMS $\geq 3$, or if the $\mathrm{MDI}<70$. The cutoff values for the NMS and the MDI were chosen to identify infants who would be targeted for rehabilitation services.

Data analysis. Statistical analysis was performed using Stata 5 (Stata Corp., College Station, TX). The predictor variables were the individual cytokine levels, and the main outcome variables were the ratios of lactate/choline and NAA/choline in the two brain regions, and neurodevelopmental outcome. Multivariable linear regression was used to test the association of cytokine levels with brain metabolite ratios while controlling for the day of life the blood sample was obtained and the MR study was performed. This association was presented as scatterplot graph without the adjustment. Spearman rank test was used to correlate the metabolite ratios with the MRI scores. Mann-Whitney $U$ tests and Fisher's exact tests were used to compare the clinical characteristics in the normal and abnormal outcome groups, and Wilcoxon matched-pairs signed-ranks tests were used to compare outcome measures at 12 and $30 \mathrm{mo}$ of age. Logistic regression was used to compare levels of cytokines in the normal and abnormal outcome groups while controlling for the day of life the blood sample was taken.

\section{RESULTS}

Subject selection. The 72 newborns enrolled in the cytokine study did not differ from the other newborns in the parent study (Fig. 1) with regard to MRI scores or neurodevelopmental outcome (all $p>0.5$ ). Although blood samples could not be located for 10 newborns enrolled in the cytokine study, these newborns had a similar distribution of injury on MRI compared with the 62 newborns whose blood spots were analyzed (all $p$ $>0.2$ ).

Infant and maternal characteristics. The 62 singleton newborns had a mean gestational age (GA) of $39.2 \mathrm{wk}$ (SD of 1.8 wk), mean birth weight of $3265 \mathrm{~g}$ (SD of $607 \mathrm{~g}$ ), and 40 were male. Maternal age (mean, $29.4 \pm$ SD 4 y) and ethnic origin were representative of the San Francisco Bay Area population: 27 Caucasian, 14 Asian/Pacific Islander, 12 Hispanic, 6 African-American, 2 Native American, and 1 mixed. Less than $10 \%$ of mothers $(6 / 62)$ had clinical suspicion of placental infection at birth, defined as maternal fever or leukocytosis, fetal tachycardia, and foul smell of uterus contents. The cytokine levels of the six newborns born to mothers with clinical chorioamnionitis were not significantly different from those without chorioamnionitis (all $p>0.1$, Mann-Whitney $U$ test). Ten placentas were available for histopathologic evaluation, of which three showed histologic chorioamnionitis. Only one of these three mothers showed clinical signs of chorioamnionitis as well. The majority (59/62) of infants received antibiotic treatment in the neonatal period, and one infant had neonatal sepsis proven by positive blood culture. Two mothers received steroid treatment for asthma during labor. The cytokine levels of the neonates who had seizures did not significantly differ from the levels measured in neonates without seizures, and 
none of the cytokines were associated with seizure severity (all $p>0.05)$.

Cytokines. Blood was collected for the cytokine analysis on median of $2 \mathrm{~d}$ of life (range, 1-8 d). Only one newborn had blood drawn after $8 \mathrm{~d}$ (on d 17); removing this newborn did not alter the results of subsequent analyses. The cytokine concentrations did not correlate with the day of life the blood was drawn $(p>0.1)$. The cytokine levels of the 12 neonates who received blood transfusion before their heel-stick samples were obtained did not significantly differ from the levels measured in the rest of the cohort $(p>0.6)$. The cytokines significantly correlated with each other, except IL-13 (Table 1).

MR data. Lactate/choline increased significantly with IL$1 \beta$, IL-6, IL- 8 , and TNF- $\alpha$ in the DGN but not in the WS zone, adjusting for the day of life the blood was drawn and the MR was performed (Table 2). The association remained significant without the adjustments (Fig. 3). IL-9, IL-12, and IL-13 were not associated with lactate/choline in either region. The measured cytokines were not associated with NAA/choline (Table 2). Similarly, the cytokine levels were not associated with the structural MRI scores (all $p>0.05$ ). However, structural MRI scores were significantly correlated with lactate/choline and NAA/choline in each respective region $(p<0.05)$.

Neurodevelopment. Of the 54 children whose neurodevelopment was assessed at 30 mo of age, 20 had abnormal outcome (Table 3): 3 were deceased due to complications of NE before reaching 6 mo of age, 4 had isolated cognitive impairment (MDI $<65$ in all; NMS $=1$ in two, NMS $=2$ in two), 5 had isolated neuromotor impairment (MDI $>80$ in all; NMS $=3$ in three and NMS $=5$ in two children), and 8 had mixed cognitive and motor abnormalities (MDI $\leq 50$ in all; NMS $=3$ in one, NMS $=5$ in seven). Of the 8 children lost to follow-up at 30 mo of age, 5 showed normal and 1 abnormal neurodevelopment at the 12-mo exam and 2 were not assessed. Overall, there was a decrease in median MDI scores from 12 to 30 mo but not in median neuromotor scores (Table 4). Specifically, seven children with normal outcome at 12 mo declined in status: three had isolated motor and four had isolated cognitive abnormalities at $30 \mathrm{mo}$. Two children with isolated motor impairment at 12 mo showed no impairment at $30 \mathrm{mo}$. Of the 10 children with mixed abnormalities at $12 \mathrm{mo}, 1 \mathrm{had}$ isolated cognitive, 1 had isolated motor impairment, and 8 still had mixed abnormalities at 30 mo. None of the children with mixed abnormalities at 12 mo were later diagnosed as normal.

Children with abnormal 30-mo outcome had more severe $\mathrm{NE}$, and were more likely to have neonatal seizures and to have been delivered by cesarean section than children with normal outcome (Table 3 ).

The one neonate with proven neonatal sepsis had abnormal outcome. His cytokine levels were in the lower range of the abnormal outcome group, and removing him from the analysis did not alter the results.

Neonatal levels of IL- $1 \beta$, IL-6, and IL-8 were significantly higher in children with abnormal outcome at 30 mo compared with those with normal neurodevelopment (Table 5). Conversely, IL-12 concentrations were lower in neonates with later abnormal outcome.

\section{DISCUSSION}

In term NE, elevations in neonatal serum inflammatory cytokines were associated with impaired cerebral oxidative metabolism in the neonatal period, and with abnormal neurodevelopmental outcome at 30 mo of age. This is consistent with our previous observation in this cohort that neonatal levels of cytokines were elevated in children with abnormal neuromotor outcome at 12 mo of age (3).

Similarly, others have found that levels of IL- 6 and IL-8 in the cerebrospinal fluid (CSF) of term newborns correlate with the degree of encephalopathy, and IL-6 levels correlate with outcome (1). Our findings are supported by a recent study of term newborns with NE that showed high plasma levels of IL-6 and high CSF levels of IL- 6 and TNF- $\alpha$ compared with controls. The high CSF/plasma ratios for these cytokines suggest that they are produced in the CNS in addition to their transport through the blood-brain barrier (12). Term infants born to mothers with clinical chorioamnionitis reached highest plasma concentrations of IL-6, IL-8, and RANTES when they presented with NE and/or seizures (2).

Cytokines may act as a final common pathway for CNS injury initiated by a variety of insults, including hypoxiaischemia, infection, reperfusion, and excitotoxins. Whether inflammatory cytokines are directly pathogenetic in neonatal brain injury or are a result of the brain injury itself is not yet understood. Studies in neonatal rodent models show that hypoxia-ischemia stimulates IL- $1 \beta$, IL- 6 , and TNF- $\alpha$ and chemokine gene expression (13) and IL- $1 \beta$ and IL- 6 bioactive protein production (14) in the CNS, and that cytokines aggravate ischemic brain injury (15). Increase of inflammatory proteins is accompanied by accumulation of neutrophils in the damaged area, activation of microglia/macrophages, lymphocytes, and astrocytes, which may persist for days after the

Table 1. Correlation coefficients between neonatal cytokine levels

\begin{tabular}{|c|c|c|c|c|c|c|c|}
\hline Cytokines & IL- $1 \beta$ & IL-6 & IL-8 & IL-9 & IL-12 & IL-13 & TNF- $\alpha$ \\
\hline IL- $1 \beta$ & 1 & & & & & & \\
\hline IL-6 & $0.9167 *$ & 1 & & & & & \\
\hline IL-8 & $0.8613^{*}$ & $0.9064 *$ & 1 & & & & \\
\hline IL-12 & $-0.2881 \dagger$ & $-0.2633 \dagger$ & $-0.2592 \dagger$ & $-0.2741 \dagger$ & 1 & & \\
\hline IL-13 & 0.0762 & 0.0768 & 0.0305 & 0.043 & -0.1589 & 1 & \\
\hline TNF- $\alpha$ & $0.8774 *$ & $0.8492 *$ & $0.7854 *$ & $0.7897 *$ & -0.2053 & 0.0559 & 1 \\
\hline
\end{tabular}

Correlations are marked as $* p<0.0001$ or $\dagger p<0.05$.

Statistical comparisons based on pairwise correlation tests. 
Table 2. Correlation of neonatal cytokine levels with cerebral metabolite ratios assessed by proton-MRS

\begin{tabular}{|c|c|c|c|c|c|c|c|c|}
\hline \multirow[b]{2}{*}{ Cytokines } & \multicolumn{2}{|c|}{$\begin{array}{l}\text { Lactate/choline } \\
\text { Deep gray nuclei }\end{array}$} & \multicolumn{2}{|c|}{$\begin{array}{c}\text { Lactate/choline } \\
\text { Watershed/cortex }\end{array}$} & \multicolumn{2}{|c|}{$\begin{array}{c}\text { NAA/choline } \\
\text { Deep gray nuclei }\end{array}$} & \multicolumn{2}{|c|}{$\begin{array}{c}\text { NAA/choline } \\
\text { Watershed/cortex }\end{array}$} \\
\hline & Coef* & $p$ Value & Coef* & $p$ Value & Coef* & $p$ Value & Coef* & $p$ Value \\
\hline IL-6 & 0.0019 & 0.005 & 0.0013 & 0.2 & -0.0005 & 0.3 & 0 & 0.9 \\
\hline IL-8 & 0.0033 & 0.007 & 0.0025 & 0.2 & -0.0004 & 0.6 & 0.0002 & 0.9 \\
\hline IL-12 & -0.0046 & 0.3 & -0.0105 & 0.2 & 0.0039 & 0.2 & 0.0038 & 0.5 \\
\hline
\end{tabular}

Statistical comparisons based on linear regression while controlled for day of life MR spectroscopy was done and blood was drawn.

* Multivariable linear regression coefficients express change in cerebral metabolites per unit increase in cytokine levels.
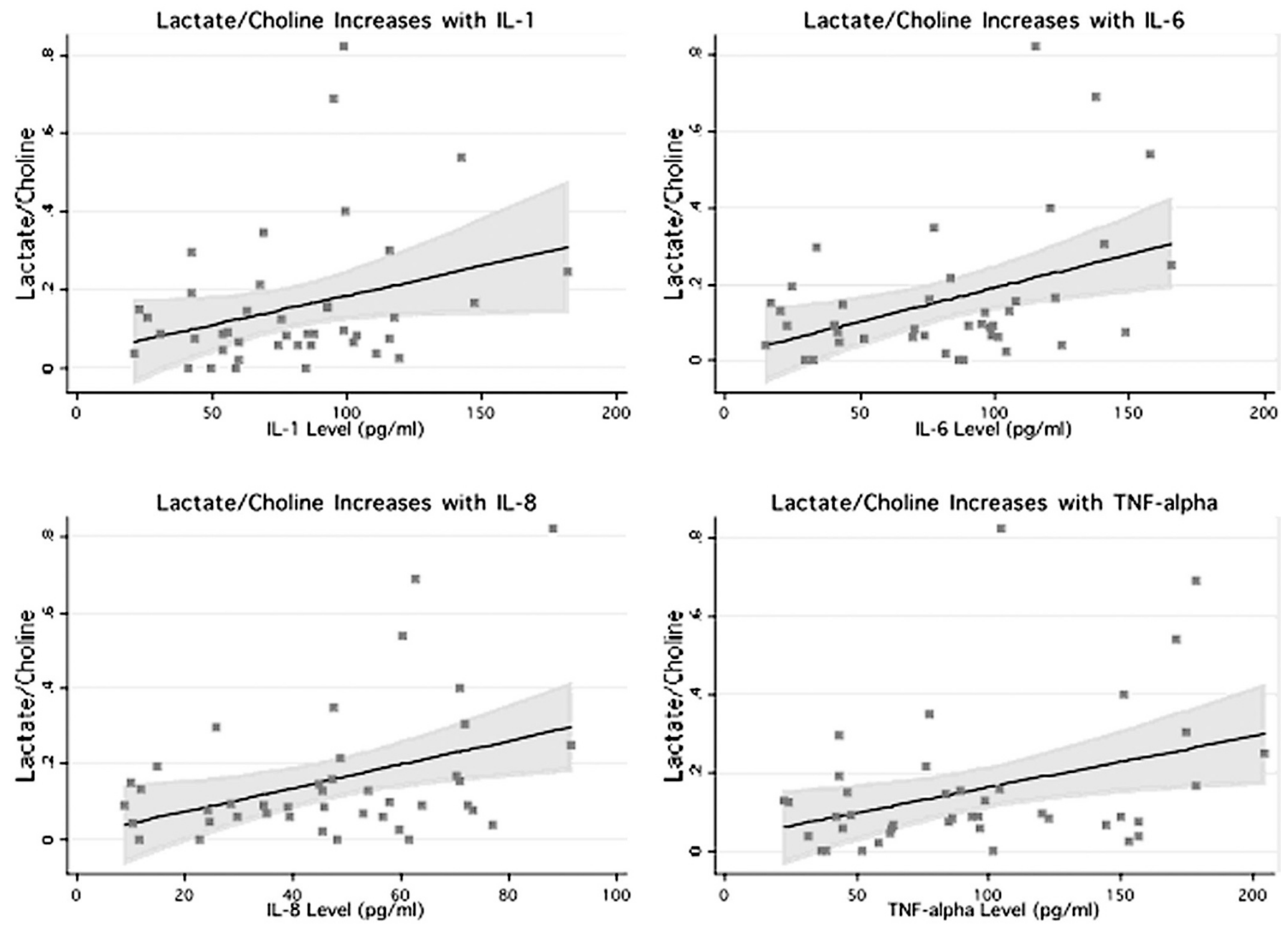

Figure 3. Association between neonatal serum cytokines and cerebral lactate/choline in the DGN. In univariate analysis, the lactate/choline increases with IL-1 $\beta$ $(p=0.05)$, IL-6 $(p=0.007)$ IL-8 $(p=0.01)$ and TNF- $\alpha(p=0.01)$. The shaded areas represent $95 \%$ confidence interval.

insult implicating a chronic state of inflammation (16). Cytokines also stimulate microglia and astrocytes to produce other cytokines, chemokines, nitric oxide, excitatory amino acids, cyclooxygenase, and reactive oxygen species $(17,18)$, which are particularly detrimental to the immature brain due to the enhanced vulnerability of maturing cells (19-21). Recent in vitro studies suggest that cytokines impair glutamate transporter function in astrocytes (22) and in oligodendrocytes (23), thus potentiating glutamate-mediated oxidative stress. Cytokines inhibit the differentiation and proliferation of oligodendrocyte precursors, which affects active myelination and may lead to white matter injury $(24,25)$. Together, these data sug- gest several potential mechanisms through which the cytokine signaling may lead to or exacerbate neonatal brain injury.

To our knowledge, this is the first study exploring the association of systemic inflammatory markers with anaerobic brain metabolism in human newborns after hypoxia-ischemia. In bacterial meningitis animal models, when anaerobic metabolism also occurs in the CNS, TNF- $\alpha$ and IL- $1 \beta$ correlate with CSF lactate levels (26), and TNF- $\alpha$ increases lactic acidosis in the CSF by reducing cerebral oxygen uptake and cerebral blood flow (27). The association of cytokines with oxidative stress is further supported by our observation that proinflammatory cytokines were associated with elevated lactate in the 
Table 3. Neonatal characteristics of children with normal and abnormal neurodevelopmental outcome at $30 \mathrm{mo}$

\begin{tabular}{|c|c|c|c|}
\hline & $\begin{array}{l}\text { Normal outcome } \\
\quad(n=34)\end{array}$ & $\begin{array}{c}\text { Abnormal } \\
\text { outcome }(n=20)\end{array}$ & $\begin{array}{c}p \\
\text { Value }\end{array}$ \\
\hline Male gender, n (\%) & $21(61 \%)$ & $15(75 \%)$ & 0.2 \\
\hline $\begin{array}{l}\text { Gestational age at delivery } \\
(\mathrm{wk})^{*}\end{array}$ & $39.5(38.9-40)$ & $38.7(37.7-39.7)$ & 0.13 \\
\hline Birth weight $(\mathrm{g})^{*}$ & $3270(3140-3435)$ & $3077(2744-3410)$ & 0.2 \\
\hline Cesarean section, n (\%) & $13(38 \%)$ & $13(58 \%)$ & 0.05 \\
\hline Umbilical artery $\mathrm{pH}^{*}$ & $7.07(6.85-7.3)$ & $7.02(6.83-7.15)$ & 0.5 \\
\hline Apgar score at $5 \mathrm{~min} *$ & $5.3(2-8)$ & $4.05(2-7)$ & 0.02 \\
\hline ECMO treatment, n (\%) & $1(3 \%)$ & $2(10 \%)$ & 0.3 \\
\hline $\begin{array}{l}\text { Meconium staining at birth, } \\
\mathrm{n}(\%)\end{array}$ & $20(59 \%)$ & $11(55 \%)$ & 0.06 \\
\hline Neonatal seizures, n (\%) & $7(21 \%)$ & $16(80 \%)$ & 0.0001 \\
\hline Encephalopathy score $\dagger$ & $3(0-6)$ & $6(2-6)$ & 0.0001 \\
\hline SNAP-PE score $\dagger$ & $23(11-38)$ & $27.5(15-57)$ & 0.008 \\
\hline Neonatal sepsis, n (\%) & 0 & 1 & 0.6 \\
\hline $\begin{array}{l}\text { Clinical chorioamnionitis, } \\
\mathrm{n}(\%)\end{array}$ & 6 & 0 & 0.06 \\
\hline $\begin{array}{l}\text { Age at heel-stick sample } \\
\text { (d) } \dagger\end{array}$ & $2(1-17)$ & $2(1-8)$ & 0.4 \\
\hline Age at MRI $(d) \dagger$ & $6(2-11)$ & $6.5(2-16)$ & 0.2 \\
\hline
\end{tabular}

Data are reported as $*$ mean $(95 \% \mathrm{CI})$ or $\dagger$ median (range). Statistical comparisons based on Mann-Whitney $U$ tests and Fisher's exact tests.

DGN but not in the WS zone. MRI studies of term newborns with NE show the DGN as the most severely injured area $(8,28,29)$, which corresponds to the spatial distribution of lactate measured by ${ }^{1} \mathrm{H}$-MRS $(9,30)$. The measured cytokines were not associated with structural abnormalities detected by MRI. It is possible that early neonatal MR scans merely are not sensitive enough to reveal mild structural injury.

The presence of neonatal seizures in term encephalopathic newborns is a reliable indicator of poor neurologic outcome $(31,32)$. As we have shown previously, increasing neonatal seizure severity was independently associated with structural injury and increased lactate/choline in the deep gray matter and the watershed zones (33). Cytokine levels were not associated with the severity of neonatal seizures, making it unlikely that the association of cytokines with impaired cerebral metabolism was confounded by the presence of seizures.

Our finding that elevated neonatal cytokines are associated with neurodevelopmental outcome is consistent with the findings of Nelson et al. (4) of higher levels of IL-1 $\beta$, IL-8, IL-9, TNF- $\alpha$, and RANTES in mostly term newborns who later developed cerebral palsy $(\mathrm{CP})$. However, in very premature infants the neonatal cytokine levels did not distinguish children

Table 4. Outcome of children $(n=54)$ assessed at 12 mo and 30

\begin{tabular}{|c|c|c|c|}
\hline Outcome & $12 \mathrm{mo}$ & $30 \mathrm{mo}$ & $p$ Value \\
\hline Neuromotor score* & $1(0-6)$ & $2(0-6)$ & 0.4 \\
\hline Cognitive score (MDI)* & $92(<50-120)$ & $84(<50-121)$ & 0.004 \\
\hline Motor abnormalities $\dagger$ & $2(4 \%)$ & $5(9 \%)$ & 0.25 \\
\hline Cognitive abnormalities $\dagger$ & 0 & $4(7 \%)$ & 0.04 \\
\hline Mixed abnormalities $\dagger$ & $10(19 \%)$ & $8(15 \%)$ & 0.1 \\
\hline Abnormal outcome & 15 & 20 & 0.09 \\
\hline Normal outcome & 39 & 34 & \\
\hline
\end{tabular}

Data are reported as *median (range) or $\uparrow$ number (percent). Statistical comparisons based on Wilcoxon matched-pairs signed-ranks tests.
Table 5. Neonatal cytokine levels of children with normal and abnormal neurodevelopmental outcome at 30 mo of age

\begin{tabular}{llll}
\hline Cytokine & \multicolumn{1}{c}{ Normal outcome $(n=34)$} & \multicolumn{1}{c}{ Abnormal outcome $(n=20)$} & $\begin{array}{c}p \\
\text { Value }\end{array}$ \\
\hline IL-1 $\beta$ & $59.8(46.1-88.1 ; 23.1-182.1)$ & $86.9(71.8-100.3 ; 36.6-147.5)$ & 0.04 \\
IL-6 & $71.9(40.4-95.3 ; 17-165.7)$ & $88.5(61.9-118.9 ; 33.6-158)$ & 0.03 \\
IL-8 & $41.6(24.1-56.7 ; 8.6-91.5)$ & $55.9(43.2-68.2 ; 15.7-88.1)$ & 0.01 \\
IL-9 & $25.45(17.3-43.2 ; 4.7-90)$ & $34.9(24.5-65 ; 6.9-85.6)$ & 0.1 \\
IL-12 & $11.9(9.7-16.4 ; 6.6-32.1)$ & $10.8(6.5-13.6 ; 4.8-18.6)$ & 0.03 \\
IL-13 & $23.4(19.6-31.6 ; 13.7-50.5)$ & $29.8(26.1-35.1 ; 14.6-49.7)$ & 0.1 \\
TNF- $\alpha$ & $71.2(43.2-101.6 ; 21.9-204.3)$ & $91.1(60.2-140.1 ; 33.2-178.4)$ & 0.1 \\
\hline
\end{tabular}

Cytokine levels are reported in $\mathrm{pg} / \mathrm{mL}$ as median (interquartile range; total range). Statistical comparisons based on logistic regression while controlling for day of life the blood sample was taken.

with CP from the control group (34). A recent study of 78 bio-markers measured in term and preterm infants later developing $\mathrm{CP}$ showed that the pattern of inflammatory markers differs in the two populations (35), suggesting that the pathways responsible for brain injury change with maturity. Our previous study of the current cohort at 12 mo of age showed that children with abnormal neuromotor outcome had elevated neonatal levels of IL-6, IL-8, and IL-9 compared with children with normal motor development (3). Our current study at 30 mo of age found an association of neonatal inflammatory markers with neurodevelopment, incorporating cognitive measures in addition to the motor outcome, as NE may result in impairments in each of these realms.

Although the accuracy and reliability of measuring a spectrum of substances in archived neonatal blood samples has been established earlier $(3,4)$, a shortcoming of this method is that it provides only a snapshot of the inflammatory cascade at the time of the blood draw. The wide time window of the blood draw (1-8d) and the MR studies (2-16 d) is a limitation of the current study, although this measurement biased our results toward the null hypothesis. Given this measurement bias, we may have underestimated or overlooked an association between serum cytokines and cerebral metabolism or neurodevelopmental outcome. Given the number of cytokines measured, it is important to note the consistency of association of the cytokine levels with lactate/choline in the DGN and not in the WS region. Due to the large overlap in the distribution of cytokine levels between the normal and abnormal outcome groups, these inflammatory markers, in isolation, are not strong prognostic predictors of neurodevelopmental outcome. Further investigations with serial bio-markers and MRI measures are needed to assess the relationship of the pro- and antiinflammatory markers to the timing of injury and how these markers are interrelated and change over time.

In a cohort study, we are unable to prove a causal link between the inflammatory environment, $\mathrm{NE}$, and subsequent neurodevelopmental impairment. The relationship of these factors may be distinct from systemic neonatal infection or chorioamnionitis. Further studies are needed to explore if modulating this pathway in the term human newborn will improve cognitive and motor outcomes.

Acknowledgments. The authors thank the Genetic Disease Branch of the California Department of Health Services for providing the archived blood spots for analysis, Dr. Benjamin 
Dickens at the Immunochemistry Laboratory at George Washington University, Washington, DC, for analyzing the blood spot samples, Dr. Giovanna Spinella at the National Institutes of Health and the Pediatric Clinical Research Center at UCSF for their continued support, and Dr. Yvonne Wu at UCSF for reviewing the manuscript.

\section{REFERENCES}

1. Savman K, Blennow M, Gustafson K, Tarkowski E, Hagberg H 1998 Cytokine response in cerebrospinal fluid after birth asphyxia. Pediatr Res 43:746-751

2. Shalak LF, Laptook AR, Jafri HS, Ramilo O, Perlman JM 2002 Clinical chorioamnionitis, elevated cytokines, and brain injury in term infants. Pediatrics 110:673-680

3. Foster-Barber A, Dickens B, Ferriero DM 2001 Human perinatal asphyxia: correlation of neonatal cytokines with MRI and outcome. Dev Neurosci 23:213-218

4. Nelson KB, Dambrosia JM, Grether JK, Phillips TM 1998 Neonatal cytokines and coagulation factors in children with cerebral palsy. Ann Neurol 44:665-675

5. Phillips TM, Krum JM 1998 Recycling immunoaffinity chromatography for multiple analyte analysis in biological samples. J Chromatog B Biomed Sci Appl 715:55-63

6. Barkovich AJ, Hajnal BL, Vigneron D, Sola A, Partridge JC, Allen F, Ferriero DM 1998 Prediction of neuromotor outcome in perinatal asphyxia: evaluation of MR scoring systems. AJNR Am J Neuroradiol 19:143-149

7. Novotny E, Ashwal S, Shevell M 1998 Proton magnetic resonance spectroscopy: an emerging technology in pediatric neurology research. Pediatr Res 44:1-10

8. McQuillen PS, Ferriero DM 2004 Selective vulnerability in the developing central nervous system. Pediatr Neurol 30:227-235

9. Barkovich AJ, Baranski K, Vigneron D, Partridge JC, Hallam DK, Hajnal BL, Ferriero DM 1999 Proton MR spectroscopy for the evaluation of brain injury in asphyxiated, term neonates. AJNR Am J Neuroradiol 20:1399-1405

10. Hajnal BL, Sahebkar-Moghaddam F, Barnwell AJ, Barkovich AJ, Ferriero DM 1999 Early prediction of neurologic outcome after perinatal depression. Pediatr Neurol 21:788-793

11. Bayley N 1993 The Bayley Scales of Infant Development II. New York Psychological Corporation, New York

12. Silveira RC, Procianoy RS 2003 Interleukin-6 and tumor necrosis factor-alpha levels in plasma and cerebrospinal fluid of term newborn infants with hypoxic-ischemic encephalopathy. J Pediatr 143:625-629

13. Szaflarski J, Burtrum D, Silverstein FS 1995 Cerebral hypoxia-ischemia stimulates cytokine gene expression in perinatal rats. Stroke 26:1093-1100

14. Hagberg H, Gilland E, Bona E, Hanson LA, Hahin-Zoric M, Blennow M, Holst M, McRae A, Soder O 1996 Enhanced expression of interleukin (IL)-1 and IL-6 messenger RNA and bioactive protein after hypoxia-ischemia in neonatal rats. Pediatr Res 40:603-609

15. Stroemer RP, Rothwell NJ 1998 Exacerbation of ischemic brain damage by localized striatal injection of interleukin-1beta in the rat. J Cereb Blood Flow Metab 18:833839
16. Bona E, Andersson A, Blomgren K, Gilland E, Puka-Sundvall M, Gustafson K, Hagberg H 1999 Chemokine and inflammatory cell response to hypoxia-ischemia in immature rats. Pediatr Res 45:500-509

17. Toulmond S, Parnet P, Linthorst AC 1996 When cytokines get on your nerves: cytokine networks and CNS pathologies. Trends Neurosci 19:409-410

18. Cowell RM, Xu H, Galasso JM, Silverstein FS 2002 Hypoxic-ischemic injury induces macrophage inflammatory protein-1alpha expression in immature rat brain. Stroke 33:795-801

19. Gabryel B, Trzeciak HI 2001 Role of astrocytes in pathogenesis of ischemic brain injury. Neurotox Res 3:205-221

20. Davies CA, Loddick SA, Toulmond S, Stroemer RP, Hunt J, Rothwell NJ 1999 The progression and topographic distribution of interleukin-1beta expression after permanent middle cerebral artery occlusion in the rat. J Cereb Blood Flow Metab 19:87-98

21. Volpe JJ 1997 Brain injury in the premature infant-from pathogenesis to prevention. Brain Dev 19:519-534

22. Liao SL, Chen CJ 2001 Differential effects of cytokines and redox potential on glutamate uptake in rat cortical glial cultures. Neurosci Lett 299:113-116

23. Fern R, Moller T 2000 Rapid ischemic cell death in immature oligodendrocytes: a fatal glutamate release feedback loop. J Neurosci 20:34-42

24. Kinney HC, Back SA 1998 Human oligodendroglial development: relationship to periventricular leukomalacia. Semin Pediatr Neurol 5:180-189

25. Pang Y, Cai Z, Rhodes PG 2003 Disturbance of oligodendrocyte development, hypomyelination and white matter injury in the neonatal rat brain after intracerebral injection of lipopolysaccharide. Brain Res Dev Brain Res 140:205-214

26. Guerra-Romero L, Tureen JH, Fournier MA, Makrides V, Tauber MG 1993 Amino acids in cerebrospinal and brain interstitial fluid in experimental pneumococcal meningitis. Pediatr Res 33:510-513

27. Tureen J 1995 Effect of recombinant human tumor necrosis factor-alpha on cerebral oxygen uptake, cerebrospinal fluid lactate, and cerebral blood flow in the rabbit: role of nitric oxide. J Clin Invest 95:1086-1091

28. Barkovich AJ $1992 \mathrm{MR}$ and CT evaluation of profound neonatal and infantile asphyxia. AJNR Am J Neuroradiol 13:959-975

29. Roland EH, Poskitt K, Rodriguez E, Lupton BA, Hill A 1998 Perinatal hypoxic-ischemic thalamic injury: clinical features and neuroimaging. Ann Neurol 44:161-166

30. Penrice J, Cady EB, Lorek A, Wylezinska M, Amess PN, Aldridge RF, Stewart A, Wyatt JS, Reynolds EO 1996 Proton magnetic resonance spectroscopy of the brain in normal preterm and term infants, and early changes after perinatal hypoxia-ischemia. Pediatr Res 40:6-14

31. Bergman I, Painter MJ, Hirsch RP, Crumrine PK, David R 1983 Outcome in neonates with convulsions treated in an intensive care unit. Ann Neurol 14:642-647

32. Robertson C, Finer N 1985 Term infants with hypoxic-ischemic encephalopathy: outcome at 3.5 years. Dev Med Child Neurol 27:473-484

33. Miller SP, Weiss J, Barnwell A, Ferriero DM, Latal-Hajnal B, Ferrer-Rogers A, Newton N, Partridge JC, Glidden DV, Vigneron DB, Barkovich AJ 2002 Seizure-associated brain injury in term newborns with perinatal asphyxia. Neurology 58:542-548

34. Nelson KB, Grether JK, Dambrosia JM, Walsh E, Kohler S, Satyanarayana G, Nelson PG, Dickens BF, Phillips TM 2003 Neonatal cytokines and cerebral palsy in very preterm infants. Pediatr Res 53:600-607

35. Kaukola T, Satyaraj E, Patel DD, Tchernev VT, Grimwade BG, Kingsmore SF, Koskela P, Tammela O, Vainionpaa L, Pihko H, Aarimaa T, Hallman M 2004 Cerebral palsy is characterized by protein mediators in cord serum. Ann Neurol 55:186-194 